\title{
Long-Term Follow-Up of 1,064-nm Picosecond-Domain Neodymium:Yttrium-Aluminum-Garnet Laser Treatment for Acquired Bilateral Nevus of Ota-Like Macules
}

\author{
So Young Kim \\ Jiho Park ${ }^{2}$ \\ Heesu Kim² \\ Sung Bin $\mathrm{Cho}^{2,3}$
}

'So Young Kim Dermatology Clinic, Seoul, Korea
2Department of Dermatology and Cutaneous
Biology Research Center, International St. Mary's
Hospital, Catholic Kwandong University College of
Medicine, Incheon, Korea
${ }^{3}$ Kangskin Sillim Dermatology Clinic, Seoul, Korea

Received November 25, 2017

Accepted November 28, 2017

\footnotetext{
Correspondence

Sung Bin Cho

Department of Dermatology and Cutaneous

Biology Research Center, International St. Mary's Hospital, Catholic Kwandong University College of Medicine, 25 Simgok-ro, Seo-gu, Incheon 22711, Korea

Tel.: +82-32-290-3141

Fax: +82-32-290-3142

E-mail: drsbchođggmail.com

(C) Korean Society for Laser Medicine and Surgery

(c) This is an open access article distributed under the terms of the Creative Commons Attribution NonCommercial License (http://creativecommons.org/ licenses/by-nc/4.0) which permits unrestricted noncommercial use, distribution, and reproduction in any medium, provided the original work is properly cited.
}

Acquired bilateral nevus of Ota-like macules (ABNOM) is a common dermal lesion of melanotic hyperpigmentation in Asians. To treat ABNOM, various nanosecond-domain lasers, including Q-switched 694$\mathrm{nm}$ ruby, 1,064-nm neodymium:yttrium-aluminum-garnet (Nd:YAG), and $755-\mathrm{nm}$ alexandrite lasers, have been used. In this report, we treated a patient with ABNOM using 1,064-nm nanosecond-domain and picosecond-domain Nd:YAG lasers. At 3 months after one session of 1,064-nm nanosecond-domain Nd:YAG laser treatment, ABNOM lesions were partially improved. Then, an additional session of 1,064-nm picosecond-domain Nd:YAG laser treatment was delivered to ABNOM lesions. The patient presented nearly complete improvement thereof with no remarkable side effects or recurrence over the duration of 18 months. Picosecond-domain lasers were deemed to generate faster photothermal reactions, more extensive photoacoustic effects, and less injury to adjacent structures than nanosecond-domain lasers. We suggest that 1,064-nm picosecond-domain $\mathrm{Nd}$ :YAG laser can be effectively and safely used for treating pigmented lesions in the dermis, particularly ABNOM, in Asian patients.

\section{Key words}

Acquired bilateral nevus of Ota-like macules; Picosecond; Nanosecond; Neodymium:yttrium-aluminum-garnet laser 


\section{INTRODUCTION}

Acquired bilateral nevus of Ota-like macules (ABNOM), also named Hori Nevus, was first described by Hori et al. in 1984. ${ }^{1}$ Clinically, ABNOM is characterized by multiple speckled blue-brown and/or slate-gray macules bilaterally on the malar region or less commonly on the forehead, upper eyelids, and nose. ${ }^{1-3}$ Skin specimens of ABNOM histologically exhibit irregularly shaped, bipolar melanocytes dispersed throughout the papillary and mid dermis, particularly in the subpapillary dermis. ${ }^{2}$

Among various treatment modalities for ABNOM, nanosecond-domain lasers have been most widely utilized, and include Q-switched 694-nm ruby, 1,064-nm neodymium:yttrium-aluminum-garnet (Nd:YAG), and 755$\mathrm{nm}$ alexandrite lasers. ${ }^{2-6}$ The treatment settings for nanosecond-domain lasers in regards to wavelength, beam size, laser fluence, intervals, and the number of treatment sessions, however, have not yet been optimized. ${ }^{2-6}$ Our study group previously evaluated 15 Korean patients with ABNOM who were treated with 1,064-nm Q-switched Nd:YAG laser. ${ }^{6}$ Therein, laser treatment was delivered at a fluence of 2.2-2.6 J/ $\mathrm{cm}^{2}$ using a 6-mm spot size on the entire face, followed by an additional pass at a fluence of 4-6 J/cm² using a 4-mm spot size on the ABNOM lesions, at 1-2-week intervals. Although most of the participants exhibited remarkable clinical improvement, the median number of treatment sessions was 11, and the minimum
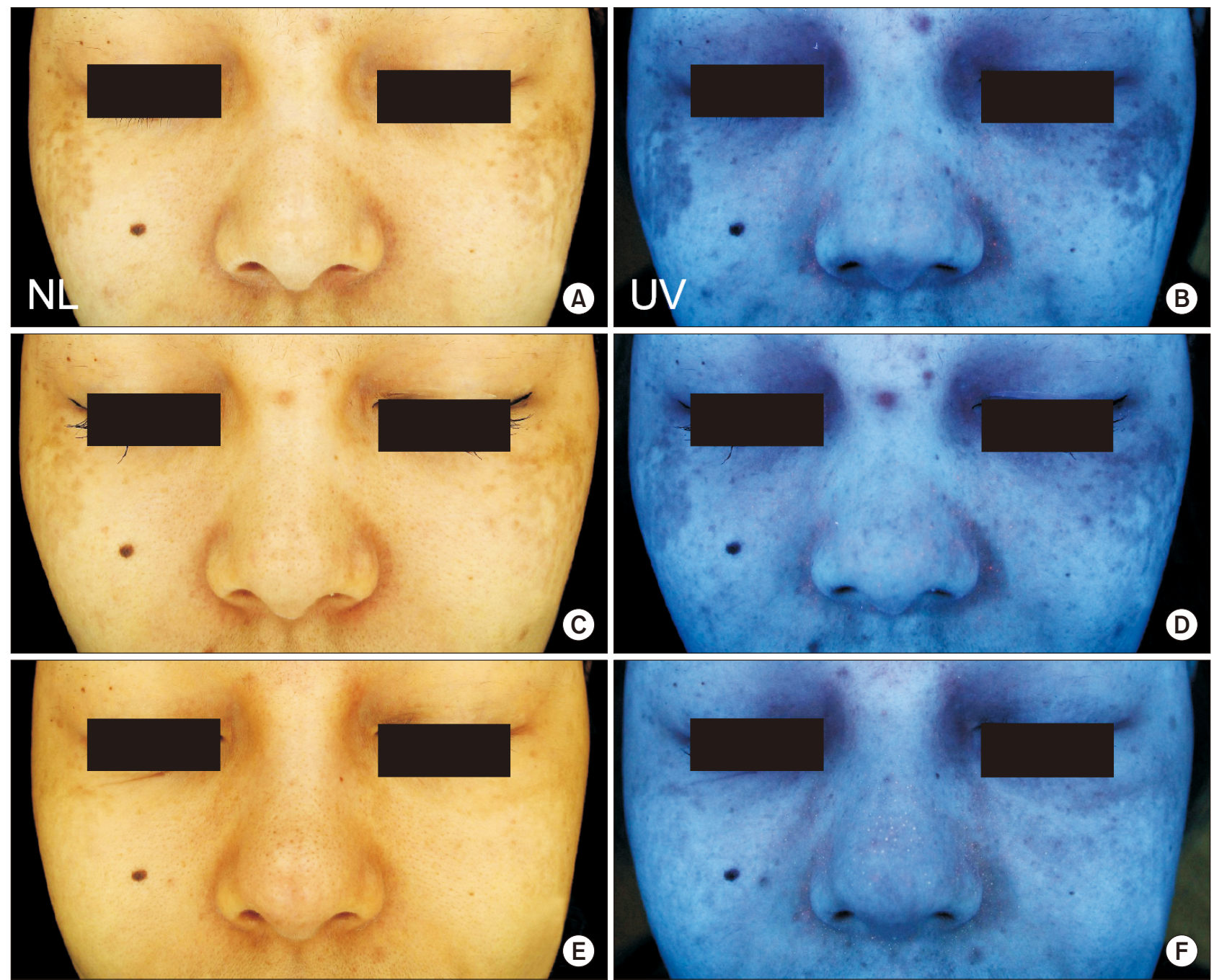

Fig. 1. Photographs of a 31-year-old, Chinese female with acquired bilateral nevus of Ota-like macules (ABNOM) (A, B) at baseline, (C, D) 3 months after a single session of 1,064-nm nanosecond-domain neodymium:yttrium-aluminum-garnet (Nd:YAG) laser treatment, and (E, F) 18 months after a single session of 1,064-nm picosecond-domain Nd:YAG laser treatment. Photographs were taken under (A, C, E) normal (NL) and (B, D, F) ultraviolet (UV) light exposure using an image tool (Janus ${ }^{\circledR}$; PSI CO., Ltd., Seoul, Korea). 
number of sessions at which clinical improvement became noticeable was $4.6 .^{6}$

In this report, we treated a patient with ABNOM using 1,064-nm nanosecond-domain and picosecond-domain $\mathrm{Nd}$ :YAG lasers. At 3 months after one session of 1,064$\mathrm{nm}$ nanosecond-domain Nd:YAG laser treatment, an additional session of 1,064-nm picosecond-domain Nd:YAG laser treatment was delivered to ABNOM lesions. Thereafter, the patient presented nearly complete improvement in the ABNOM lesions with no remarkable side effects or recurrence over the duration of 18 months.

\section{CASE REPORT}

A 31-year-old, Chinese woman visited our clinic presenting with confluent, round to oval, brown macules on both malar areas. The patient presented no remarkable treatment history for facial pigmentation, such as topical retinoid or bleaching agent applications, chemical skin resurfacing procedures, and laser or light treatments using intense pulsed light, long-pulsed lasers, or nanosecondor picosecond-domain lasers, within the last six months. The patient was clinically diagnosed with ABNOM (Fig. 1A, 2A, 3A), and was scheduled to undergo laser treatment.

After obtaining written informed consent, the patient's face was gently cleansed with a mild soap, and topical anesthetic cream leutectic mixture of 2.5\% lidocaine $\mathrm{HCl}$ and $2.5 \%$ prilocaine, EMLA; Astra Pharmaceuticals, Westborough, MA, USA) was applied for 1 hour. Then the patient was treated with a single session of 1,064-nm nanosecond-domain $\mathrm{Nd}$ :YAG laser treatment (Spectra XT; Lutronic Corp., Goyang, Koreal at treatment settings of a 7-mm spot size, a $1.8-\mathrm{J} / \mathrm{cm}^{2}$ fluence in a pulse-topulse mode, and a total of approximately 2,000 shots on
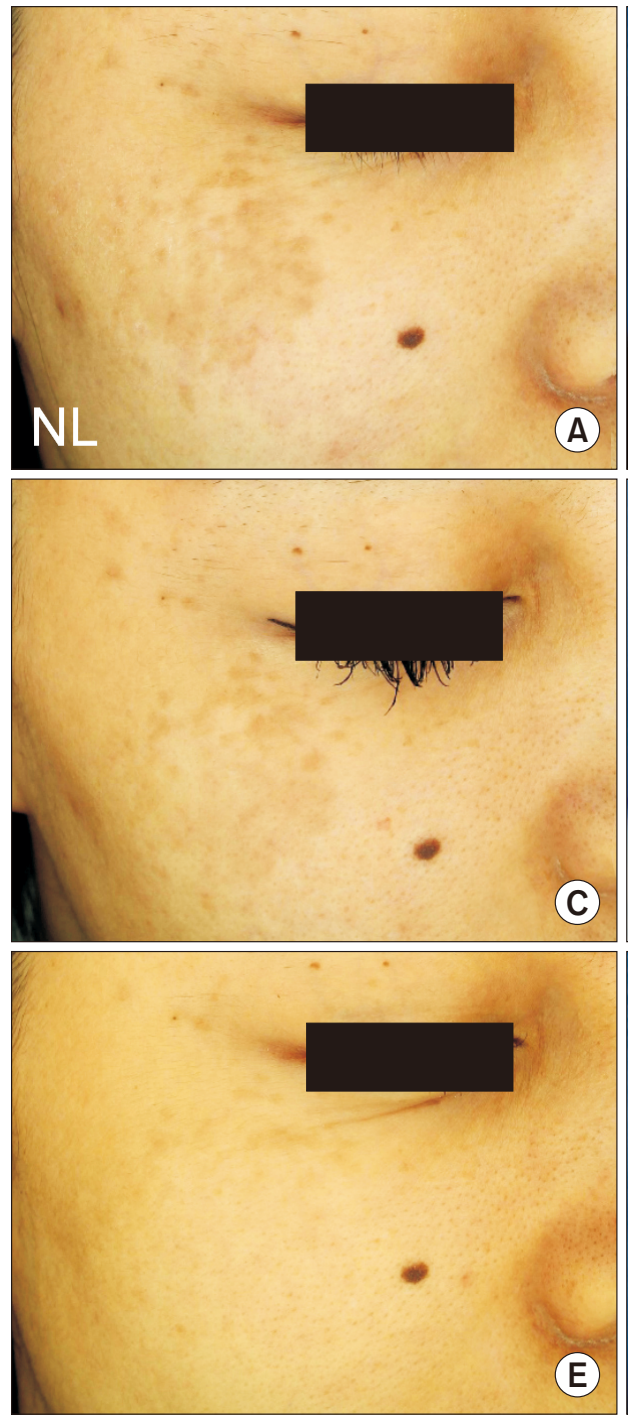
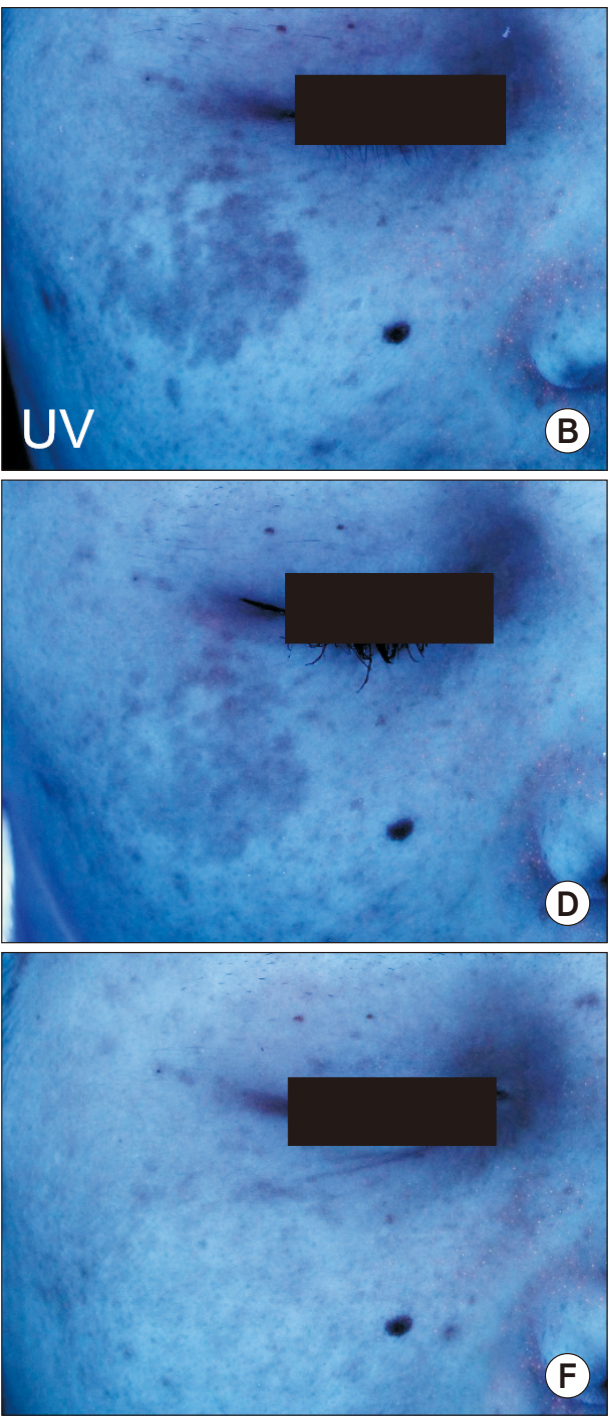

Fig. 2. Right oblique view of the patient with ABNOM (A, B) at baseline, (C, D) 3 months after a single session of 1,064-nm nanoseconddomain Nd:YAG laser treatment, and (E, F) 18 months after a single session of 1,064-nm picosecond-domain Nd:YAG laser treatment. Photographs were taken under (A, C, E) NL and (B, D, F) UV light exposure. 

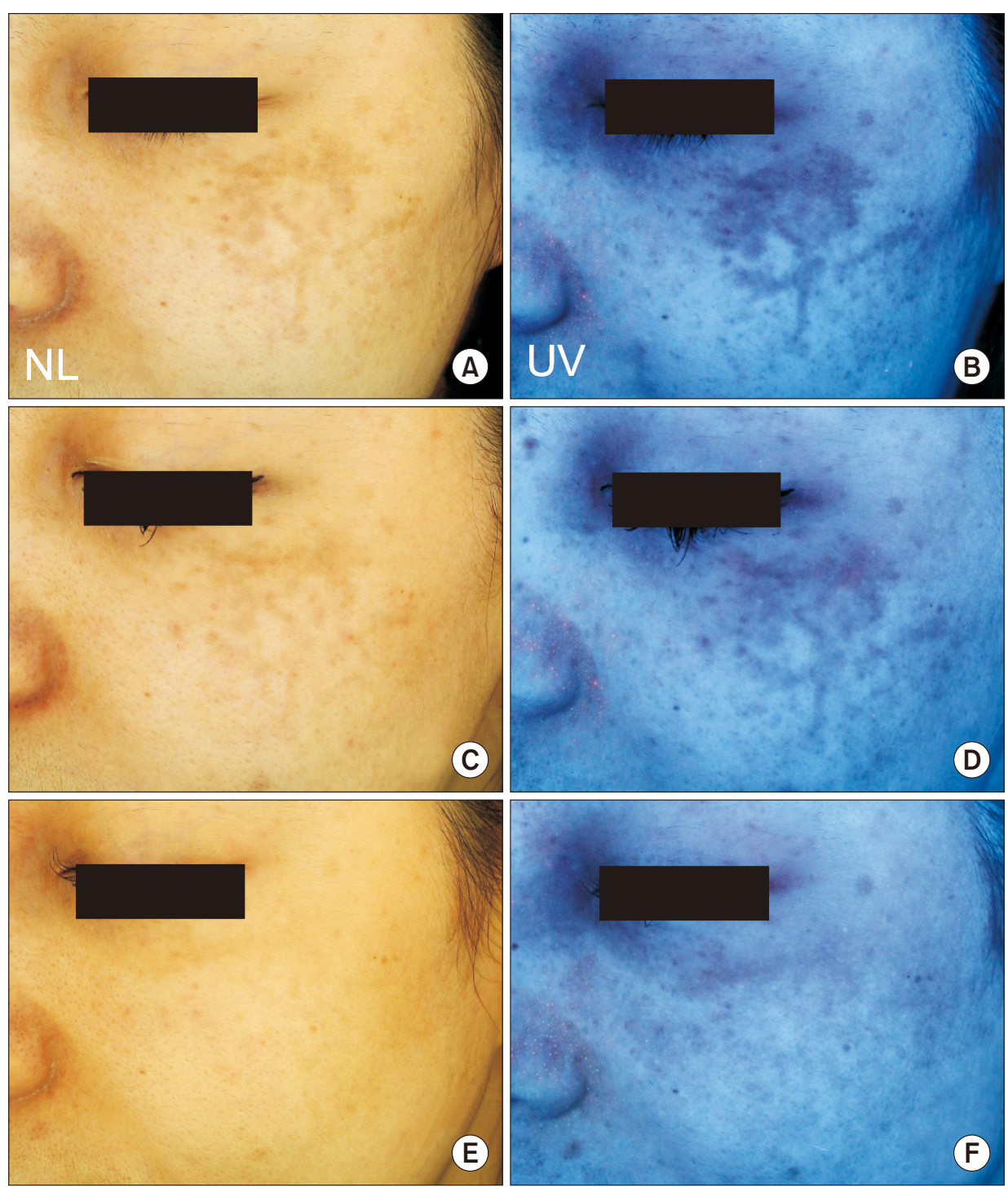

C
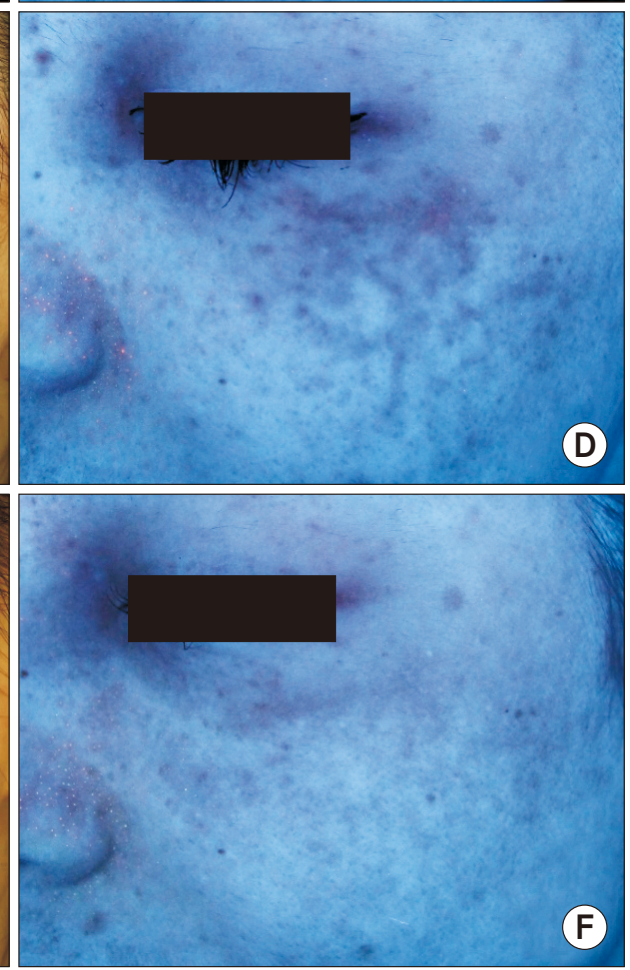

Fig. 3. Left oblique view of the patient with $\mathrm{ABNOM}(\mathrm{A}, \mathrm{B})$ at baseline, $(\mathrm{C}$, D) 3 months after a single session of 1,064-nm nanosecond-domain Nd:YAG laser treatment, and (E, F) 18 months after a single session of 1,064-nm picosecond-domain Nd:YAG laser treatment. Photographs were taken under (A, C, E) NL and $(\mathrm{B}, \mathrm{D}, \mathrm{F}) \mathrm{UV}$ light exposure. the entire face. Additional pulses were delivered on the ABNOM lesions at the spot size of $4 \mathrm{~mm}$ and the fluence of $5.4 \mathrm{~J} / \mathrm{cm}^{2}$ in a single pulse mode over three passes. The treated areas were cooled with icepacks; no prophylactic systemic or topical corticosteroids were prescribed. The patient was recommended to apply sunscreen and to avoid topical bleaching and retinoid agents. At 3 months after 1,064-nm nanosecond-domain Nd:YAG laser treatment, the ABNOM lesions had slightly improved, compared with baseline (Fig. 1B, 2B, 3B).

Thereafter, a single session of 1,064-nm picoseconddomain Nd:YAG laser treatment (PICOPLUS; Lutronic Corp.) was performed at the treatment settings of a 7 -mm spot size, a $0.6-\mathrm{J} / \mathrm{cm}^{2}$ fluence in a single pulse mode, and a total of approximately 2,000 shots on the entire face. Additional pulses were delivered on the residual
ABNOM lesions at the spot size of $4 \mathrm{~mm}$ and the fluence of $2.4 \mathrm{~J} / \mathrm{cm}^{2}$ in a single pulse mode over 3 passes. The treated areas were cooled with icepacks, and no prophylactic systemic or topical corticosteroids were prescribed. The patient was recommended to apply sunscreen and to avoid topical bleaching and retinoid agents. The patient reported that the pigmented lesions had remarkably improved over 3 months. At 18 months after 1,064-nm picosecond-domain $\mathrm{Nd}$ :YAG laser treatment, the patient presented nearly complete improvement of the ABNOM lesions without worsening or recurrence thereof (Fig. 1C, 2C, 3C). Furthermore, no major side effects, including petechial, bleeding, oozing, scaling, crusting, prolonged post-therapy erythema, postinflammatory hyperpigmentation or hypopigmentation, and scarring, were encountered. 


\section{DISCUSSION}

ABNOM is a dermal melanocytic hyperpigmentation that exhibits histological features similar to those of nevus of Ota. ' Selectively destroying target pigmented chromophores, nanosecond-domain laser devices have been accepted as the treatment of choice for nevus of Ota. Qswitched lasers, including Q-switched ruby, Nd:YAG, and alexandrite lasers, have also been used for treating $A B$ $\mathrm{NOM}^{3-5}$ Despite the similarities between ABNOM and nevus of Ota, the therapeutic outcomes after nanoseconddomain laser treatment thereof are superior in nevus of Ota lesions than in ABNOM lesions; moreover, the risk of developing post-laser treatment hyperpigmentation is higher in ABNOM lesions than in nevus of Ota. ${ }^{2}$ Furthermore, although $Q$-switched laser treatments generally result in satisfactory clinical outcomes, the treatment settings for treating ABNOM with nanosecond-domain lasers have not yet been optimized. ${ }^{2-6}$

Picosecond-domain lasers are reportedly more efficacious in removing pigment in the form of tattoos than nanosecond-domain lasers. ${ }^{7-9}$ Moreover, the risk of side effects is lower in picosecond-domain lasers, compared with nanosecond-domain lasers. ${ }^{7.8}$ Theoretically, particles of smaller size among target chromophores after successive treatment would be better reactive to subsequent picosecond-domain laser treatment than nanoseconddomain laser treatment. ${ }^{8}$ Our recent simulation study using tissue-mimicking phantoms demonstrated that picosecond-domain laser treatments disintegrated tattoo pigments more homogeneously than nanoseconddomain treatments. ${ }^{9}$ Moreover, the additional delivery of laser pulses at the picosecond-pulse duration further broke down tattoo pigments that were pretreated with nanosecond-domain lasers. ${ }^{9}$ In the study, high-speed cinematography revealed that successive laser pulses at the picosecond-pulse duration generated greater degrees of acoustic wave reflection and propagation over hundreds of microseconds to a few milliseconds, compared with nanosecond-pulse duration. ${ }^{9}$ A randomized, controlled trial, however, concluded that picosecond-domain lasers do not provide better effects on clearing tattoos than nanosecond-domain lasers, although they elicit less pain during treatment. ${ }^{10}$

In this report, we demonstrated near-complete clinical improvement in pigmented lesions after treatments with 1,064-nm nanosecond-domain and picoseconddomain Nd:YAG lasers in a Chinese patient with ABNOM. Although our patient presented clinical improvement thereof after a single session of nanosecond-domain laser treatment, the additional picosecond-domain laser treatment resulted in marked clearance of residual $A B-$ NOM lesions. The laser pulses at the picosecond-pulse duration were deemed to further destroy target chromophores initially disintegrated by the laser pulses at the nanosecond-pulse duration in our patient.

In conclusion, we suggest that 1,064-nm picoseconddomain Nd:YAG lasers can be effectively and safely used for treating pigmented lesions in the dermis, particularly ABNOM, in Asian patients. Notwithstanding, further prospective, controlled investigations should be followed to compare the efficacy and safety of nanosecond- and picosecond-domain lasers and to optimize the treatment settings thereof for ABNOM.

\section{ACKNOWLEDGEMENTS}

We would like to thank Anthony Thomas Milliken, ELS (Editing Synthase, Seoul, Korea) for his help with the editing of this manuscript. This work was supported by a R\&D Program for Forest Science Technology (Project No. 2017033A00-1719-BA01) funded by the Korea Forest Service (Korea Forestry Promotion Institute).

\section{REFERENCES}

1. Hori Y, Kawashima M, Oohara K, Kukita A. Acquired, bilateral nevus of Ota-like macules. J Am Acad Dermatol 1984;10:961-4.

2. Park JM, Tsao H, Tsao S. Acquired bilateral nevus of Ota-like macules (Hori nevus): etiologic and therapeutic considerations. J Am Acad Dermatol 2009;61:88-93.

3. Kunachak S, Leelaudomlipi P, Sirikulchayanonta V. Q-Switched ruby laser therapy of acquired bilateral nevus of Ota-like macules. Dermatol Surg 1999;25:938-41.

4. Suh DH, Han KH, Chung JH. Clinical use of the Q-switched $\mathrm{Nd}: Y A G$ laser for the treatment of acquired bilateral nevus of Ota-like macules (ABNOMs) in Koreans. J Dermatolog Treat 2001;12:163-6.

5. Lam AY, Wong DS, Lam LK, Ho WS, Chan HH. A retrospective study on the efficacy and complications of $\mathrm{Q}$-switched alexandrite laser in the treatment of acquired bilateral nevus of Otalike macules. Dermatol Surg 2001;27:937-41.

6. Cho SB, Park SJ, Kim MJ, Bu TS. Treatment of acquired bilateral nevus of Ota-like macules (Hori's nevus) using 1064nm Q-switched Nd:YAG laser with low fluence. Int J Dermatol 2009;48:1308-12.

7. Ross V, Naseef G, Lin G, Kelly M, Michaud N, Flotte TJ, et al. Comparison of responses of tattoos to picosecond and nanosecond Q-switched neodymium: YAG lasers. Arch Dermatol 1998;134:167-71. 
8. Ross EV. The picosecond revolution and laser tattoo treatments: are shorter pulses really better? Br J Dermatol 2017;176:299300.

9. Ahn KJ, Zheng Z, Kwon TR, Kim BJ, Lee HS, Cho SB. Pattern analysis of laser-tattoo interactions for picosecond- and nanosecond-domain 1,064-nm neodymium-doped yttriumaluminum-garnet lasers in tissue-mimicking phantom. Sci
Rep 2017;7:1533.

10. Pinto F, Große-Büning S, Karsai S, Weiß C, Bäumler W, Hammes $S$, et al. Neodymium-doped yttrium aluminium garnet (Nd:YAG) 1064-nm picosecond laser vs. Nd:YAG 1064-nm nanosecond laser in tattoo removal: a randomized controlled single-blind clinical trial. Br J Dermatol 2017;176:457-64. 